\title{
The disappearance of dichoptically presented real and subjective contours
}

\author{
DIANE F. HALPERN \\ California State University, San Bernardino, California \\ and \\ JOEL S. WARM \\ University of Cincinnati, Cincinnati, Ohio
}

\begin{abstract}
The fragmentation characteristics of luminous figures set off by real or subjective contours were studied under dichoptic viewing conditions. Dichoptically viewed subjective contours fragmented in a manner quantitatively and qualitatively similar to that previously obtained with binocular viewing. The number of fragmentations for real contour analogues increased substantially under dichoptic relative to binocular conditions. These results are considered to support the view that subjective contours arise primarily from central processes within the perceptual system.
\end{abstract}

In recent years, there has been considerable interest in a phenomenon known as subjective contours. The term "subjective contours" refers to the appearance of contours where none physically exist, that is, in the absence of local luminance discontinuities. Two examples of such stimuli are presented in the upper right-hand column of Figure 1. Observers report the presence of a square and a circle in these figures, yet contours are objectively present only along the edges of the inducing areas.

There have been numerous attempts to identify the mechanisms that fabricate contours in homogeneous areas of a visual field. One category of hypotheses posits cognitive explanations in which observers are viewed as active organisms who seek to create and maintain a meaningful and stable percept by utilizing depth cues, closure, and figure ground relationships (e.g., Rock \& Anson, 1979). A second line of investigation is concerned primarily with the physiological correlates of subjective contour perception. According to this position, subjective contours are perceived when portions of the stimulus display activate neural feature detectors, brightness contrast mechanisms, and/or spatial frequency analyzers (e.g., Day \& Jory, 1980; Smith \& Over, 1979). Multivariate explanations combining depth cues, closure, and brightness contrast have also been proposed (Halpern, 1981; Halpern, Salzman, Harrison, \& Widaman, 1983; Salzman \& Halpern, 1982). For a review of the literature, see Halpern and Salzman (1983).

The authors thank William N. Dember and Richard Honeck for their valuable comments on this manuscript and David Meder for technical assistance. Reprint requests should be sent either to Diane F. Halpern, Department of Psychology, California State University, San Bernardino, 5500 State College Parkway, San Bernardino, CA 92407, or to Joel S. Warm, Department of Psychology, University of Cincinnati, Cincinnati, OH 45221.

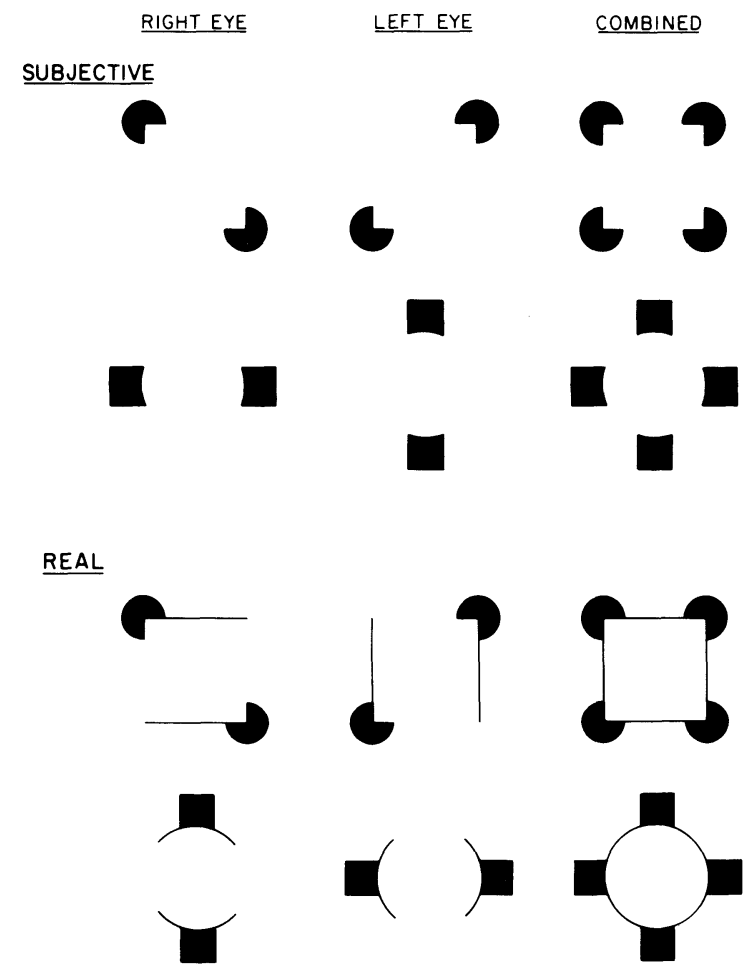

Figure 1. Stimulus elements presented to right and left eye and their combination.

An underlying question for much of the research on subjective contours is the degree to which central and peripheral mechanisms contribute to their perception. The luminous design technique described by McKinney $(1963,1966)$ provides an appropriate tool for this investigation. He found that when luminous drawings are observed under dim illumination, they fragment, 
with parts disappearing and reappearing in meaningful perceptual units. Similar results have been reported by other investigators (e.g., Swanston, 1979). Recently we (Halpern \& Warm, 1980) made use of the luminous design technique to attack the problem of a common origin for the perception of real and subjective contours. Subjects were asked to report the fragmentation of the real and subjective contour stimuli presented in the right column of Figure 1. We found that, among real contour figures, most disappearances involved a fading of the entire figure or a portion of the contour; there were relatively few disappearances in the inducing areas. By contrast, with subjective contour figures, most of the fragmentation occurred in the physically present inducing areas. Such results have the paradoxical implication that the subjects were reporting that "what was there wasn't and what wasn't there was!" These findings led us to conclude that real and subjective contours do not share a common perceptual origin.

One way of probing the origin of subjective contour perception is with dichoptic viewing, which involves presenting each eye with a portion of a subjective contour display so that contour perception is possible only if the observer integrates the stimulus input to each eye. Previous research (Bradley, 1982; Kennedy \& Chattaway, 1975) has shown that subjective contours can arise from the integration of dichoptically presented stimulus information.

The purpose of the present study was to assess the fragmentation characteristics of dichoptically presented subjective contours in order to provide additional information for deciding among the possible origins of subjective contour perception.

\section{METHOD}

\section{Subjects}

Forty female and 40 male students from introductory psychology classes served as subjects. All had normal or corrected-to-normal vision.

\section{Design}

A mixed design, in which contour type (real and subjective) was a between-subjects factor and stimulus configuration (square and circle) was a within-subjects factor, was used.

\section{Stimuli}

Two geometric configurations, a square and a circle created with real or subjective contours, were cut from heavy black construction paper. Sheets of Polacoat polarizing film were glued to the stimuli, behind the cut portions. Film orientation was arranged so that observers wearing $90-\mathrm{deg}$ opposed polarizing lenses would receive selected portions of the stimulus to each eye. The stimulus elements visible to the right and left eyes are presented in the left-hand and middle columns, respectively, of Figure 1. The real and subjective contour patterns that result from the combination of the right- and left-eye stimulation are presented in the right-hand column of Figure 1. To keep the overall luminance differences between the figures as small as possible, double-edge real figures were used as comparitors for the subjectives. We did this because overall luminance is a critical factor in the perception of subjective contours (Dumais \& Bradley, 1976) and because overall luminance is also an important element in the fragmentation paradigm that we employed.

\section{Procedure}

Forty subjects were assigned at random to each contour condition with the restriction that there be an equal number of females and males in each condition. As a further control procedure, half of the females and males in each group viewed the stimulus components as presented in Figure 1, whereas the other half viewed the stimulus components labeled "left eye" with their right eye, and vice versa.

The subjects were seated in front of a table in a totally darkened room. A 12-in. square electroluminescent panel with uniform luminosity was presented at eye level, directly in front of the subject. Stimuli appeared as black figures on a pale green background. The luminance of the electroluminescent panel was approximately $.3426 \mathrm{~cd} / \mathrm{m}^{2}$. The stimuli subtended a visual angle of approximately $14 \mathrm{deg}$. This visual angle ensured that disappearances would not be confounded with receptor insensitivity to dim illumination associated with foveal vision. Pilot work was conducted to ensure that each eye received only the stimulus portions illustrated in Figure 1.

The subjects were dark-adapted for $5 \mathrm{~min}$ at the start of the experimental session. They were asked to wear the 90-deg opposed polarizing lenses without being informed of the dichoptic nature of the presentation. The subjects were told that when a stimulus was exposed, they would see a luminous figure glowing in the dark and that stimulus fragmentation occurs under viewing conditions such as these. The subjects were instructed to depress a telegraph key located near their preferred hand as soon as any portion of the stimulus disappeared and to keep the key depressed until it reappeared. The subjects were told that, if there was a change in the nature of the fragmentation, they should lift their finger momentarily and then depress the key again, and continue to keep it depressed for the duration of the new fragment. Keypresses were transcribed onto an EsterlineAngus cumulative recorder that kept track of the number of fragmentations that occurred during the viewing period.

Each stimulus was presented for a 3-min inspection period. Prior to the start of an inspection period, the stimulus was first uncovered until the subject could identify the configuration. This was done to ensure that each subject actually saw the entire figure before reporting disappearances. All subjects reported that the figures were clearly visible. In addition, no subject reported difficulty in fusing the stimuli, and there were no complaints of discomfort arising from "double images."

At the end of each inspection period, the subjects were provided with answer sheets containing 10 identical dotted configurations that matched the stimulus just seen. For example, if a subject had inspected a square, either subjective or real, the answer sheet contained 10 dotted squares. The subjects' task was to trace over the portion of the figure that disappeared, up to a maximum of 10 disappearances, for as many of the disappearances that could be remembered. Duplications were permitted. The tracing method of reporting disappearances has been shown to be preferable to verbal reports, which bias subjects' responses toward easily verbalized descriptions (Schuck, 1973).

\section{RESULTS}

\section{Quantitative Data}

The number of fragmentations for each 3-min viewing period was tallied for each subject. These scores were subjected to a square-root transformation (as suggested by Myers, 1979, for frequency data). An analysis of variance revealed that significantly more disappearances occurred for the real contour figures than for the subjective contour figures $[F(1,78)=4.12, p<.05]$. Neither the main effect of figure type nor the contour $x$ figure type interaction was significant.

This result is opposite to that obtained in an earlier binocular disappearance study (Halpern \& Warm, 1980), 
in which significantly more disappearances occurred for the subjective contour figures. Because these studies are identical in all respects except for the binoculardichoptic differences in stimulus presentation, the mean number of disappearances for the real and subjective contour forms from each study are presented in Table 1 for comparison.

Perusal of Table 1 reveals that the mean number of subjective contour disappearances remained approximately the same under binocular and dichoptic conditions (18.09 binocular; 16.98 dichoptic), whereas the mean number of real contour disappearances was more than 2.5 times as great under dichoptic than under binocular conditions (10.50 binocular; 28.98 dichoptic).

\section{Qualitative Data}

The qualitative data were analyzed in two parts-by eye of disappearance and by portion of stimulus fragmented. Each will be reported in turn.

Eye of disappearance. One possible outcome with dichoptically presented stimuli is that all disappearances occur in the nondominant eye. Long (1979) urged caution in the use of dichoptic viewing paradigms for the purpose of determining the locus of an effect. Long's caveat that ocular dominance be controlled in dichoptic viewing paradigms was heeded in the present study by analyzing the data for dominance effects. Eye dominance was ascertained at the start of the experiment with a simple sighting task. Of the 80 subjects employed, $52(65 \%)$ were right-eye dominant and $28(35 \%)$ were left-eye dominant. The number of disappearances that occurred for each eye was scored by assigning each stimulus tracing from each subject to one of the following categories: disappearance of stimuli presented to right eye only; disappearance of stimuli presented to left eye only; or disappearance of stimuli presented to both eyes. The disappearance of any portion of the subjective contour was also attributed to both eyes because the subjective contours were perceived only when both monocular inputs were combined. The eye-of-disappearance data were analyzed by an analysis of variance. For this analysis, contour type (real and subjective) and dominant eye (right or left) were the between- subjects factors, and eye of disappearance (right, left, and both) and form (square and circle) were the withinsubjects factors. All components of this analysis failed to obtain significance.

Portion of stimulus fragmented. To determine which portion or portions of the figures disappeared, each tracing was assigned to one of the following disappearance categories: entire figure, inducing area or portion of an inducing area only, contour or portions of a contour only, or a combination of inducing area and contour. Because of the high variability in disappearance rates among subjects, category scores were then converted to proportions. Each subject's qualitative disappearance measure was expressed as four proportions, one of each disappearance category. Because proportion data necessarily sum to 1 for each subject, and therefore are not independent, an analysis of variance was computed on the first three categories only. For this analysis, contour type (real and subjective) was a betweensubjects factor, and form (square and circle) and disappearance category (entire figure, inducing area only, and contour only) were the within-subjects factors. A significant main effect was found for disappearance category $[F(2,156)=17.08, p<.001]$. The disappearance category $\mathrm{x}$ contour type interaction was also statistically significant $[F(2,156)=9.03, p<.001]$. All other sources of variance failed to reach significance. The disappearance category $\mathrm{x}$ contour type interaction is presented graphically in Figure 2.

\section{DISCUSSION}

The disappearance characteristics of dichoptically presented subjective contours are essentially similar to those previously obtained with binocular viewing. These results suggest a central locus for the subjective contour phenomenon. Visual perception involves a continuum of processes anchored at one end by retinal effects and at the other by higher cognitive effects. If subjective contour perception is mediated by events that are largely cognitive (i.e., closer to the cognitive endpoint of the continuum than the retinal endpoint), then presenting the stimulus elements dichoptically should have little effect on the processes involved in their perception. The qualitative disappearance data lends further support to this hypothesis. Among the subjective contour forms, slightly more than half of the disappearances occurred only in the physically present inducing areas. This compares

Table 1

Mean Number of Disappearances (MND) of Two Geometric Forms Displayed With Real and Subjective Contours During a 3-Min Viewing Period

\begin{tabular}{|c|c|c|c|c|c|}
\hline \multirow[b]{3}{*}{ Contour Type } & \multicolumn{4}{|c|}{ Viewing Condition } & \multirow[b]{3}{*}{ Mean } \\
\hline & \multicolumn{2}{|c|}{ Binocular $(N=36)^{*}$} & \multicolumn{2}{|c|}{ Dichoptic $(\mathrm{N}=80)$} & \\
\hline & MND & SD & MND & SD & \\
\hline Subjective & & & & & \\
\hline Square & 18.06 & 14.28 & 16.20 & 10.69 & 17.13 \\
\hline Circle & 18.11 & 9.37 & 17.75 & 11.57 & 17.93 \\
\hline Real & & & & & \\
\hline Square & 11.44 & 7.97 & 27.85 & 11.13 & 19.65 \\
\hline Circle & 9.56 & 6.13 & 30.10 & 12.12 & 19.83 \\
\hline Mean & 14.29 & & 22.98 & & \\
\hline
\end{tabular}

*Data from Halpern and Warm (1980). 


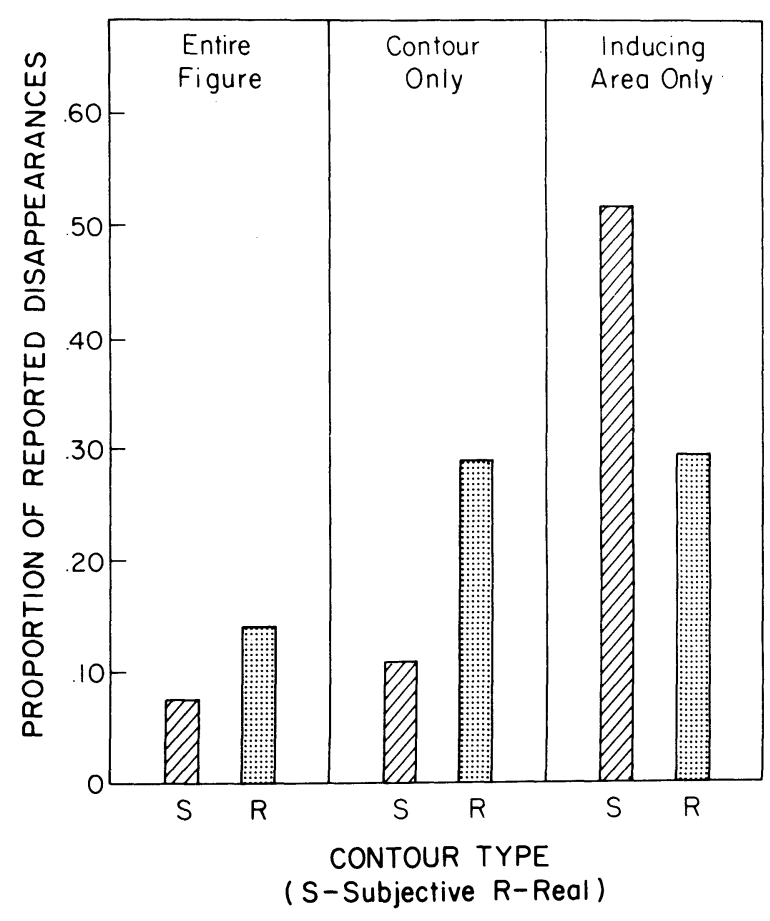

Figure 2. Mean proportion of reported disappearances for subjective and real contour stimuli as a function of portion of stimulus display that disappeared.

favorably with the slightly more than $40 \%$-inducing-area disappearances found with binocular viewing of subjective contour figures (Halpern \& Warm, 1980). Thus, dichoptic presentation had little effect on the disappearance of subjective contours relative to their disappearance with binocular viewing.

A persisting question that has emerged from the disappearance studies with subjective contours is: Why do most disappearances occur in the physically present portions of the stimulus display and not in the contours fabricated somewhere in the observer's visual system? It seems that when observers supply the contours, they are more resistant to fragmentation than when the contours are physically present in the stimulus display. In the jargon of information processing, top-down contours are less prone to disappearance than bottom-up ones. Disappearance paradigms traditionally have been employed to allow the inference of neural feature analyzers in the human visual system (e.g., Weisstein, 1969). If subjective contour portions of the stimulus rely primarily on processes unrelated to the activity of feature analyzers, then these contours might be expected to show a low fragmentation frequency relative to the physically present portions. This is exactly what has happened.

Results obtained with real contour stimuli show considerable discrepancies between dichoptic and binocular conditions. When dichoptic viewing is compared with binocular viewing, it can be seen that the number of fragmentations almost tripled for real contour stimuli. The increase in number of disappearances may have been mediated by binocular rivalry caused by the endpoints of the line segments. If the increase in disappearance rate for real contour figures presented dichoptically relative to the disappearance rate for those presented binocularly is due to rivalry, then the failure of subjective contour figures to mimic this increase points to etiological differences for these two contour types. Bradley (1982) provided further evidence against a rivalry explanation. He found that dichoptic presentations of subjective contours "did not result in binocular rivalry or contralateral suppression of the contours" (p. 86).

In addition to disparities between real and subjective contours in the rate of fading, qualitative disparities in regard to the loci of disappearances were also noted. Among subjective figures, most of the fragmentations were seen in the inducing areas only. This result, obtained with dichoptic viewing, clearly replicates our earlier findings with binocular viewing. It adds to the reliability of the notion that, when witnessing the fading of subjective stimuli, subjects in effect report that the subjectively defined portions of the figures (i.e., those portions having no physical correlate) are visible at times when the objectively real figural portions are not. Recently, Gellatly (1980) reported an identical finding. Using backward visual masking, he was able to mask the inducing areas of a subjective contour figure so that subjects reported that the figure was perceived when the inducing areas were not phenomenally present.

The data obtained in this experiment and in previous research using the luminance design paradigm strongly suggest a central locus for subjective contour perception. The fact that the disappearance characteristics of contours that are fabricated by the "mind's eye" are quantitatively and qualitatively different from those of real contours, which arise from retinal-level stimulation, supports the hypothesis that they result from etiologically different mechanisms.

\section{REFERENCES}

Bradley, D. R. (1982). Binocular rivalry of real vs. subjective contours. Perception \& Psychophysics, 32, 85-87.

DAY, R. H., \& JoRY, M. K. (1980). A note on a second stage in the formation of illusory contours. Perception \& Psychophysics, 27, 89-91.

Dumais, S. T., \& Bradley, D. R. (1976). The effects of illumination level and retinal size on the apparent strength of subjective contours. Perception \& Psychophysics, 19, 339-345.

Gellatly, A. R. (1980). Perception of an illusory triangle with masked inducing figure. Perception, 9, 599-602.

HALPERN, D. F. (1981). The determinants of illusory-contour perception. Perception, 10, 199-213.

Halpe Rn, D. F., \& Salzman, B. (1983). The multiple determination of illusory contours: 1. A review. Perception, 12, 281-291.

Halpern, D. F., Salzman, B., Harrison, W., \& Widaman, K. (1983). The multiple determination of illusory contours: 2 . An empirical investigation. Perception, 12, 293-303.

HALPE RN, D. F., \& WARM, J. S. (1980). The disappearance of real and subjective contours. Perception \& Psychophysics, 28, 229-235.

Kennedy, J. M., \& Chattaway, D. (1975). Subjective contours, binocular and movement phenomena. Italian Journal of Psychology, 11, 353-367.

Long, G. M. (1979). The dichoptic viewing paradigm: Do the eyes have it? Psychological Bulletin, 86, 391-403.

McKinney, J. P. (1963). Disappearance of luminous designs. Science, 140, 403-404.

MCKINNEY, J. P. (1966). Verbal meaning and perceptual stability. Canadian Journal of Psychology, 20, 237-242.

MYERS, J. (1979). Fundamentals of experimental design (3rd ed.). Boston: Allyn \& Bacon.

Rock, I., \& ANSon, R. (1979). Illusory contours as the solution to a problem. Perception, 8, 655-681.

Salzman, B., \& Halpern, D. F. (1982). Subjective towers: Depth relationships in multilevel subjective contours. Perceptual and Motor Skills, 55, 1247-1256.

Schuck, J. R. (1973). Factors affecting reports of fragmenting visual images. Perception \& Psychophysics, 13, 382-390.

Smith, A. T., \& Over, R. (1979). Motion aftereffect with subjective contours. Perception \& Psychophysics, 25, 95-98.

Swanston, M. T. (1979). The effects of verbal meaning and response categorisation on the fragmentation of steadily fixated patterns. Perception, 8, 635-646.

WEISSTEIN, N. (1969). What the frog's eye tells the human brain: Single cell analyzers in the human visual system. Psychological Bulletin, 72, 157-176.

(Manuscript received for publication May 17, 1984.) 